ARTÍCULO ORIGINAL

\title{
Experiencia de personas con diabetes y prediabetes sobre la metodología educativa del Programa de Intervención Nutricional de Enfermedades Crónicas, Costa Rica
}

Sonia Guzmán-Padilla ${ }^{1 *}$, Marlene Roselló-Araya² y Andrea Ramírez-Sanabria ${ }^{3}$

${ }^{1}$ Nutricionista-Investigadora, Unidad de Salud y Nutrición, Instituto Costarricense de Investigación y Enseñanza en Nutrición y Salud (INCIENSA); ${ }^{2}$ Nutricionista-Epidemióloga, Investigadora, Educadora en Diabetes, Unidad de Salud y Nutrición, INCIENSA; ${ }^{3}$ Nutricionista, Educadora en Diabetes, Pasante INCIENSA, Unidad de Salud y Nutrición, Universidad de Costa Rica, Departamento de Bioquímica. Costa Rica

\section{RESUMEN}

Introducción: La educación en diabetes se considera una estrategia efectiva para que los usuarios asuman la responsabilidad de su enfermedad y donde los servicios de salud pueden intervenir oportunamente brindando las herramientas necesarias que contribuyan al control glicémico y a la prevención o retraso de las complicaciones. Objetivo: Describir la experiencia sobre la metodología educativa de las personas con diabetes y prediabetes que participaron en el Programa de Intervención Nutricional de Enfermedades Crónicas (PINEC). Metodología: Evaluación cualitativa mediante grupos focales. Se realizó un muestreo no probabilístico por conveniencia. Participaron 21 adultos (19 con diabetes y 2 con prediabetes), quienes asistieron a las seis sesiones de PINEC. La información obtenida

\section{ABSTRACT}

Introduction: Diabetes education is considered an effective strategy for users to take responsibility for their illness and where health services can intervene in a timely manner, providing the necessary tools that contribute to glycemic control and the prevention or delay of complications. Objective: To describe the experience on the educational methodology of people with diabetes and prediabetes who took part in the PINEC. Methodology: Qualitative evaluation through focus groups. A non-probabilistic convenience sampling was done. 21 adults participated (19 with diabetes and 2 with prediabetes), who attended the six sessions of PINEC. The information obtained was analyzed with the program Ethnograph ${ }^{\circledR}$ V4.0, in the following categories: educational sessions, group
Correspondencia:

*Sonia Guzmán Padilla

E-mail: sguzmandinciensa.sa.cr
Fecha de recepción: 24-09-2018

Fecha de aceptación: 20-01-2019 Doi: 10.24875/ALAD.19000364 
se analizó con el programa Ethnograph ${ }^{\circledR}$ V4.0, en las siguientes categorías: sesiones educativas, metodología grupal, recursos didácticos y rol de los facilitadores. Resultados: Este estudio evidencia que el PINEC tuvo buena aceptación por parte de los participantes, quienes adquirieron conocimientos y mejoraron su estilo de vida. La metodología grupal, las sesiones y los recursos didácticos beneficiaron el proceso de aprendizaje; además, la persona facilitadora fue clave para propiciar la motivación en quienes participaron y así realizar cambios en su estilo de vida para el control de su enfermedad. Discusión: El PINEC fue evaluado de manera positiva, por lo que el programa se tomó como base para la atención y enseñanza de las personas con diabetes y prediabetes en el sistema de salud público de Costa Rica.

Palabras clave: Diabetes. Prediabetes. Educación terapéutica. Evaluación cualitativa. Grupos focales. methodology, didactic resources and role of facilitators. Results: This study shows that the PINEC was well accepted by the participants, who acquired knowledge and improved their lifestyle. The group methodology, the sessions and the didactic resources benefited the learning process, in addition the facilitator was key to propitiate the motivation of the participants and thus make changes in their lifestyle for the control of their disease. Discussion: The PINEC was evaluated positively, so the program was taken as a basis for the care and education of people with diabetes and prediabetes in the public health system of Costa Rica. (Rev ALAD. 2019;9:44-54)

Corresponding author: Sonia Guzmán Padilla, sguzman@inciensa.sa.cr

Key words: Diabetes. Prediabetes. Therapeutic education. Qualitative education. Focal groups.

\section{INTRODUCCIÓN}

La diabetes mellitus tipo 2 (DM2) es una enfermedad multicausal y crónica degenerativa, la prediabetes (Pre-D) es el estado que la precede. En Costa Rica, para la población de 20 años y más (según datos de la Encuesta de Factores de Riesgo Cardiovascular) la prevalencia de DM2 fue del $10.8 \%$ en el 2010, cifra que aumentó al $12.8 \%$ en el 2014; para la Pre-D, un $16.5 \%$ en el 2010 y un $20.1 \%$ en el $2014^{1,2}$.

Ambas condiciones requieren de un seguimiento a largo plazo, el cual debe contemplar alimentación saludable, realización de actividad física, tratamiento farmacológico y educación; esta última se considera indispensable para que las personas usuarias

asuman la responsabilidad de su propia enferme$\operatorname{dad}^{3,4}$. Se ha demostrado que los programas educativos estructurados dirigidos a personas con diabetes son una estrategia de intervención exitosa en la cual los servicios de salud pueden intervenir oportunamente y dar las herramientas necesarias que contribuyan al control glicémico y a la prevención o retraso de las complicaciones ${ }^{5,6}$.

La Organización Mundial de la Salud7 refiere que la educación en salud es fundamental para el control de la diabetes, por lo que la implementación de programas educativos debe ser un eje prioritario en los establecimientos de salud, con el propósito de facilitar a la persona el correcto cumplimiento del tratamiento, así como el autocontrol de su 
enfermedad; la evidencia científica ha demostrado el impacto y los beneficios de los programas educativos en diabetes ${ }^{8-13}$.

Según la Asociación Americana de Diabetes ${ }^{14}$, los programas de educación dirigidos a esta población deben definirse bajo el contexto de educación terapéutica «centrada en la persona y no en la enfermedad» y el proceso de aprendizaje basado en la formación de competencias (saber conocer, saber hacer y saber ser) $)^{15-17}$.

Por esta razón, en el 2010, el Instituto Costarricense de Investigación y Enseñanza en Nutrición y Salud (INCIENSA) y la Caja Costarricense de Seguridad Social (CCSS) crearon e implementaron el Programa de Intervención Nutricional en Enfermedades Crónicas (PINEC), el cual es una metodología de educación terapéutica grupal basada en competencias dirigida a personas con diabetes y Pre-D e integrada al tratamiento médico, cuyo fin es mejorar la capacidad de las personas usuarias para asumir el control y la responsabilidad de su propia vida con la enfermedad.

El PINEC consta de dos módulos, el básico de tres sesiones en un mes (una vez por semana) y el módulo de mantenimiento (una sesión a los dos, cuatro y seis meses), en grupos pequeños de ocho a diez personas, quienes asisten en compañía de un familiar. Además, tiene guías educativas, una para diabetes y otra para Pre-D, donde se unificaron los contenidos temáticos: generalidades de la enfermedad, alimentación y su relación con el tratamiento farmacológico, salud cardiovascular, control y reducción de peso y resolución de situaciones de la vida de las personas con diabetes y Pre- $\mathrm{D}^{18}$.

En este proceso, el profesional en nutrición se capacitó con el propósito de actualizar los conocimientos en el tratamiento de estas enfermedades y brindar las herramientas necesarias de la educación terapéutica, asumir el rol de profesional facilitador y fomentar un ambiente adecuado de aprendizaje para que la persona usuaria asuma el manejo de su enfermedad ${ }^{19}$. En las sesiones educativas, el personal facilitador utiliza una serie de técnicas didácticas como demostraciones, dramatizaciones y actividades bailables para la pausa activa, con sus propios instrumentos y materiales didácticos elaborados para el PINEC.

El programa se dirige a la población con DM o Pre-D mayor de 20 años de edad, atendidos en la consulta nutricional en los establecimientos de la CCSS, el sistema de salud pública nacional. EI PINEC entre el 2012 y el 2016 se implementó en 23 establecimiento de salud; de los 1,700 pacientes que asistieron, el $88.6 \%$ $(n=1,507)$ presentaba diabetes y el $11.4 \%(n=193)$ Pre-D; la edad promedio fue de $53 \pm 11$ y $49 \pm 9$ años, respectivamente, diferencia estadísticamente significativa ( $p$ < 0.005). Tomando en cuenta que la educación es uno de los principales factores para lograr el éxito en el control de la DM2 y la Pre-D, el objetivo de este estudio es describir la experiencia sobre la metodología educativa de las personas con diabetes y Pre-D que participaron en el PINEC.

\section{METODOLOGÍA}

\section{Tipo y población de estudio}

Se trata de un estudio cualitativo con entrevistas semiestructuradas y grupos focales. Los informantes fueron personas usuarias que completaron la intervención educativa del PINEC implementada en los establecimientos de salud de la CCSS. Se les invitó a participar de manera voluntaria por medio de llamadas telefónicas y se les informó acerca de la actividad a realizar, su propósito y su carácter de confidencialidad. Para la determinación de las personas participantes se utilizó el muestreo teórico, en el 
TABLA 1. Caracterización de los participantes del PINEC

\begin{tabular}{|l|l|}
\hline Variables & Características \\
\hline Total participantes & 21 personas (19 DM2 y 2 Pre-D) \\
\hline Sexo & 14 mujeres y 7 hombres \\
\hline Rango de edad & Entre 22 y 71 años (edad promedio: 52.4 años) \\
\hline Ocupación & Amas de casa, pensionados, secretarias, profesores, misceláneos y estudiantes \\
\hline Escolaridad & $\begin{array}{l}3 \text { primaria incompleta } \\
3 \text { primaria completa } \\
8 \text { secundaria incompleta } \\
1 \text { secundaria completa } \\
1 \text { universidad incompleta } \\
4 \text { universidad completa }\end{array}$ \\
\hline Años de diagnóstico de la enfermedad & $\begin{array}{l}\text { Entre } 1 \text { a } 23 \text { años (promedio } 6.2 \text { años) } \\
11 \text { (52.4\% menos de } 5 \text { años) }\end{array}$ \\
\hline
\end{tabular}

DM2: diabetes mellitus tipo 2; PINEC: Programa de Intervención Nutricional de Enfermedades Crónicas; Pre-D: prediabetes.

Fuente: Base de datos del Instituto Costarricense de Investigación y Enseñanza en Nutrición y Salud, $2013^{17}$.

cual no se establece un número determinado de personas, sino que se suman mientras se completa la información, hasta que esta no aporte nada nuevo (saturación teórica), este punto se alcanza cuando la información comienza a repetirse ${ }^{20}$.

Para la entrevista semiestructurada se elaboró una guía con siete preguntas clave, la cual fue validada previamente en un grupo de personas usuarias que habían finalizado la intervención educativa del Área de Salud de Coronado.

Se realizaron tres grupos focales conducidos por profesionales en nutrición (ajenos al PINEC), quienes asistieron a capacitaciones. Cada sesión de grupo tuvo una duración de 88 minutos.

\section{Análisis de datos}

La información obtenida fue transcrita literalmente y procesada en el programa Ethnograph ${ }^{\circledR} \mathrm{V} 4.0$ y, a partir del análisis de contenido de los grupos focales, se establecieron manualmente las categorías.

\section{RESULTADOS}

La población en estudio estuvo conformada por 21 informantes (19 con DM2 y 2 con Pre-D), el 67\% de los cuales eran mujeres. La edad promedio de la muestra fue de 52.4 años de edad. Respecto a la ocupación, la mayoría eran amas de casa y con los estudios de secundaria incompletos. El $52.4 \%$ tenían menos de 5 años de diagnóstico de la enfermedad, con un promedio de 6.2 años (Tabla 1).

Con base en el análisis de contenido y partiendo de que la experiencia es la «práctica prolongada que proporciona conocimiento o habilidad para hacer algo ${ }^{21}$, se estructuraron las siguientes categorías: sesiones educativas, metodología grupal, recursos didácticos y rol del personal facilitador.

\section{Experiencia de los participantes sobre las sesiones educativas}

Con respecto a las sesiones educativas, se exploró principalmente el contenido temático, el número de 
sesiones y el tiempo de cada una, entre otros parámetros.

La intervención educativa, como se mencionó, constaba de seis sesiones en las cuales se trataron temas relacionados con alimentación, medicamentos y actividad física. Al respecto, las personas participantes manifestaron que el contenido temático desarrollado en las diferentes sesiones les permitió adquirir más conocimientos, pues desconocían algunos conceptos y de otros tenían ideas erróneas.

«Aquí fue donde aprendí el porqué de algunas cosas o del todo yo no sabía» (mujer, 41 años, profesora, Coronado).

«Bueno, por ejemplo, aquí yo aprendí, a pesar de que tengo años de ser diabética, aprendí la regla 15/15, verdad y la comprobé» (mujer, 63 años, ama de casa, Coronado).

Indicaron que la educación brindada fue «necesaria» en el manejo de su patología, debido a deficiencias en el tipo de educación y atención que habían recibido previamente a su participación en PINEC.

"Son cosas que uno tal vez, al no tener conocimiento usted no las sabe y usted no las corrige... usted lo puede hacer si sabe la información, pero digámosle, una persona que no sabe, es como que no puede ver» (hombre, 22 años, estudiante, Alajuela).

La educación es un elemento indispensable para el tratamiento y manejo de la diabetes y la Pre-D, es necesario aprender a vivir con la patología, concepto que reforzaron quienes participaron.

«Porque uno tiene que saber vivir con la enfermedad, hay que aprender a vivir con la enfermedad, hay que aprender, poco a poco uno va aprendiendo» (mujer, 55 años, ama de casa, Puntarenas).
El proceso les ayudó a entender la enfermedad, pues refieren que lograron explicar, ayudar y aconsejar a otras personas con diabetes que incurrían en los mismos errores que ellos, antes de iniciar el PINEC.

«Me ayudó a mí y yo fui y se lo expliqué a mi suegra cómo tomarse el tratamiento, porque ella era otra que se lo tomaba igual que yo; entonces me ayudó a mí, yo ayudé a otra persona y eso es como una cadena» (mujer, 55 años, ama de casa, Barranca).

Todos afirmaron que les agradó participar en el programa y mencionaron que les «gusta estar acá (en el PINEC)». Expresaron que les encantan y les gustan las sesiones, y agregaron que es una opción económica y accesible al desarrollarse cerca de su comunidad. Mencionaron que este proceso educativo superó sus expectativas y lo recomendarían a otras personas.

Una característica del PINEC es que al desarrollarse varios grupos durante el año, permitió a las personas participantes reponer las sesiones a las cuales, por alguna circunstancia, no pudieron asistir.

Además, comentaron que durante las sesiones siempre se mostró interés por su participación, valoraron que el programa no tenga un enfoque evaluativo, sino que tiene el objetivo de mejorar su calidad de vida mediante la educación y que lo importante es completar todas las sesiones.

«Incluso, en una de ellas (de las sesiones) yo falté y entonces me dijeron "pero usted no vino a la cuarta (sesión), entonces véngase con el otro grupo", entonces uno dice qué bueno, hasta están previniendo y todo que uno por equis razón no venga» (hombre, 62 años, pensionado, Coronado).

En la sesión de alimentación es donde se orienta al paciente en temas como porciones de alimentos, 
cumplimiento de horarios de comida, intercambios y distribución de carbohidratos. Las personas participantes mencionaron que en esta sesión aprendieron a negociar, es decir, a intercambiar correctamente harinas, leguminosas, frutas, leche y yogurt, porque comprendieron que todos aportan la misma cantidad de carbohidratos por porción.

«Me enseñaron a comer y aprendí a comer mejor, me ha ayudado a aprender que uno tiene que desayunar, porque a veces uno se levanta y se pone a hacer otras cosas y se le olvida que tiene que desayunar» (mujer, 48 años, miscelánea, Barranca).

Manifestaron que las sesiones se llevaron a un ritmo adecuado, pues los temas no estaban sobrecargados de información, además se ajustaban a las condiciones de aprendizaje de las personas usuarias.

«El programa fue poquito a poquito, no fue que le llovieron todas las ideas» (hombre, 40 años, fabricante de muebles, Coronado).

\section{Experiencia de los participantes sobre la metodología grupal}

La categoría sobre la experiencia de la metodología grupal evaluó la aceptación de compartir con personas con las mismas características e intereses comunes. La metodología grupal tuvo gran aceptación, pues lo que más les gustó fue participar con otras personas pacientes, asimismo les permitió aprender ellas, tanto por el intercambio de experiencias como por las preguntas y dudas que el grupo externalizó. Compartir con otras personas que presentan la misma enfermedad se convirtió en un sistema de apoyo y se identificaron entre sí.

«A mí lo que me gustó fue la terapia de grupo, digamos, yo aprendo de ella (la facilitadora), de ellos (otros participantes) aprendo también» (hombre, 62 años, pensionado, Coronado).

«Porque sí aprende uno y lo ayudan a uno las charlas y este sistema de que uno se reúne, que uno oye las diferentes opiniones, uno también va agarrando como ideas para uno, verdad» (mujer, 41 años, profesora, Coronado).

La metodología grupal fue una fuente de motivación, pues les ayudó a tomar decisiones y realizar cambios en su estilo de vida, al escuchar prácticas implementadas por otras personas participantes:

«Entonces cada vez que yo le iba a echar mantequilla al pan me acordaba de ella (participante) y ya no le echaba mantequilla» (mujer, 60 años, ama de casa, Coronado).

Escuchar los logros y metas alcanzadas por otras personas del grupo les demostró que el tratamiento integral de la enfermedad funciona y que el paciente es capaz de aplicarlo si otras también lo han logrado.

"Y felicito a don Sigifredo (participante) y uno dice "vea ese, quiero ser él, bajó 18 kilos, yo también quiero bajar, yo puedo"» (hombre, 40 años, fabricante de muebles, Coronado).

Vale recalcar que las personas participantes tienen presente que estos beneficios no se obtienen de la consulta individual e incluso afirmaron que preferían la metodología grupal después de haber asistido a PINEC.

«Para mí es muy importante esto, insisto, la terapia en grupo, porque cuando uno llega aquí, no es lo mismo que lo atiendan individualmente a uno que cuando lo atienden en grupo, porque inclusive el nivel de glicémia que trae cada uno, uno oye los otros, entonces 
uno más o menos anda viendo, como anda uno verdad» (hombre, 62 años, pensionado, Coronado).

Finalmente, de acuerdo con la opinión de quienes participaron, la incorporación de los familiares en el programa fue un apoyo fundamental para sobrellevar la enfermedad, pues cuando el cambio de hábitos y prácticas se limita solamente a la persona con diabetes, sienten que se convierten en una carga para los demás.

«El apoyo familiar es importante para todos nosotros, porque si no hay el apoyo de la casa estamos mal, estamos mal» (hombre, 69 años, pensionado, Puntarenas).

\section{Experiencia de los participantes sobre los recursos didácticos}

Una parte fundamental que se consideró para implementar este programa fue el diseño y reproducción de material educativo, tales como afiches del plan de alimentación, modelos de alimentos, afiche con síntomas de hipoglucemia e hiperglucemia, afiches para la identificación de alimentos con carbohidratos y etiquetas de alimentos procesados, entre otros.

Al referirse a estos recursos didácticos utilizados en cada sesión, las personas participantes indicaron que fueron apropiados. Algunas mencionaron que se generaba un «clima de confianza», lo cual favoreció que pudieran expresarse libremente e indicaron que el periodo de pausa activa en las sesiones (en la cual se utiliza música y baile) es uno de los elementos que potencia este ambiente, pues es algo muy de compartir.

Como parte del programa, a cada paciente se le entrega el plan de alimentación individualizado. A este respecto, las personas participantes recalcaron que les gusta tener su plan de alimentación.

«Y el plan de alimentación es bueno, muy bueno, porque nos los dieron a cada persona individual, entonces eso ayuda mucho bastante» (mujer, 60 años, ama de casa, Coronado).

También reiteraron y valoraron de manera positiva el diseño del plan de alimentación, el cual les posibilita recordar «cómo vienen clasificados los alimentos y las cosas que uno tiene que comer de cada uno de los grupos» (hombre, 22 años, estudiante, Alajuela).

De forma general, quienes participaron indicaron que los materiales eran apropiados, que «estaban bien» y describieron a las ayudas visuales como «muy buenas».

Hicieron énfasis en el material de refuerzo, el cual consideraron como una herramienta útil, que les permitió repasar en caso de duda, para reforzar y afianzar conocimientos.

«Porque uno aprende mucho, como le digo, yo a veces cojo el libro (material de refuerzo) y aprende uno más» (mujer, 60 años, ama de casa, Coronado).

"Yo lo que hago cuando se me olvida algo, es que voy a buscar el folleto, yo lo busco y lo reviso» (mujer, 48 años, miscelánea, Barranca).

\section{Experiencia de los participantes sobre el rol del equipo facilitador}

El rol del equipo facilitador es de vital importancia para que la educación sea efectiva con el fin de lograr el autocuidado, por lo que esta categoría exploró sus habilidades pedagógicas y su capacidad de comunicación. 
Sobre este aspecto, las personas participantes describieron al equipo facilitador como un grupo de profesionales con un trato muy bueno, que se caracterizaron por su amabilidad y las hizo mantenerse muy bien, al no sentirse amenazadas ni juzgadas.

«El trato que dan las profesionales que vienen con nosotros son excelentes también» (hombre, 69 años, pensionado, Puntarenas).

La persona facilitadora se convirtió en un elemento que brindó motivación y contribuyó al cambio en sus estilos de vida, así como en el seguimiento de las recomendaciones del tratamiento integral de la patología.

«Porque yo cuando voy a comerme algo, me acuerdo de ella, me acuerdo, me acuerdo... ella está ahí, como detrás mío» (hombre, 62 años, pensionado, Coronado).

Una de las características de la educación terapéutica es el uso de lenguaje sencillo y concreto, con explicaciones claras y concisas de los conceptos brindados por parte del equipo facilitador, por lo que las personas participantes se vieron favorecidas en esta intervención educativa.

"Se ve que a ella (facilitador) le gusta lo que hace y se ve como ella le explica a uno todo, entonces usted no se queda con la duda, ella le explica y te integra todo, todo» (mujer, 63 años, ama de casa, Coronado)

«Aquí sí me han explicado bien [...] a ustedes yo les he entendido mucho, que la insulina es, como me enseñaron en el PINEC, la llavecita para abrir la puerta de la célula para que entre el azúcar al cuerpo» (hombre, 69 años, pensionado, Puntarenas).

Otro aspecto que resaltaron quienes participaron fue que las personas facilitadoras no utilizaron los regaños y la imposición de cambios en el estilo de vida, más bien lo que se fomenta es la atención centrada en la persona y no en la enfermedad.

«Este (programa) es el que más veo que tienen interés por el paciente» (mujer, 63 años, ama de casa, Coronado).

«No es usted tiene esto y esto, hágalo, ¿no lo hizo?, es problema suyo» (hombre, 40 años, fabricante de muebles, Coronado).

\section{DISCUSIÓN}

EI PINEC se estructuró bajo el concepto de educación terapéutica basada en competencias, por lo que las sesiones educativas, las ayudas audiovisuales, los instrumentos y el material didáctico se diseñaron considerando las necesidades y características de las personas, adaptados a las particularidades socioculturales e individuales de quienes participaron. Lo anterior facilitó el proceso educativo tanto para las personas usuarias como para el equipo de profesionales de salud. Según Hevia ${ }^{22}$, la educación en diabetes debe considerar todos los aspectos mencionados, con el fin de que las intervenciones educativas sean efectivas y las personas con diabetes adopten estilos de vida saludables.

Cabe mencionar que el objetivo de la educación en las personas con diabetes es mejorar el conocimiento y las habilidades, capacitándolas para asumir el control de la enfermedad e integrar el autocontrol en la vida cotidiana. Se debe promover la socialización y compartir experiencias que le permitan a la persona y a su familia tomar decisiones conscientes e informadas.

EI PINEC se centró en la metodología grupal, la cual parte de la premisa de que la persona aprende con 
y por el otro, donde el aprendizaje se obtiene de las experiencias individuales en relación con la enfermedad, por lo que la interacción entre sí les ayuda a no sentirse solas y las motiva hacia el autocuida$\mathrm{do}^{23}$, aspecto expresado en este estudio por quienes participaron.

Por lo tanto, los programas educativos deben ser centrados en la persona y no en la enfermedad, con actividades de «aprender haciendo» para favorecer el proceso de aprendizaje y la adquisición de estilos de vida saludables ${ }^{15}$.

Trento, et al. ${ }^{24}$ demostraron que la intervención grupal tiene un mayor efecto en la adquisición de nuevos conocimientos cuando el proceso se adapta a las necesidades y características de la persona, independientemente de la escolaridad y la edad. Además, se señala la efectividad de las intervenciones educativas como estrategia de autocuidado y control de la diabetes a corto plazo ${ }^{25}$.

Se ha identificado en varios estudios ${ }^{26-30}$ que las personas participantes comprenden y asimilan mejor la información cuando se repasan o repiten los temas, aspecto identificado como positivo por quienes participaron en el PINEC. Asimismo, el rol que ejerce el equipo de facilitadores (en el caso de este estudio el profesional de Nutrición) fue clave en el desarrollo de la confianza entre profesional y paciente y así propiciar un clima de cercanía y aceptación, para poner en práctica las recomendaciones y controlar su enfermedad.

En las actividades grupales no siempre se puede homologar el tipo de paciente, pero la persona educadora o facilitadora debe crear un clima de interacción para que este pueda expresarse libremente. Dichos profesionales deben tener habilidades pedagógicas, así como la capacidad de comunicarse, escuchar y comprender, con el propósito de obtener efectos positivos en el cambio de actitudes de las personas ${ }^{31}$.

Dado lo anterior, en el PINEC (previo a su implementación) el grupo de profesionales o facilitadores fue capacitado en la metodología grupal, en la educación terapéutica, así como en métodos y técnicas de comunicación interactiva, lo cual fue clave para el éxito del programa, pues el aprendizaje se vio favorecido por la utilización de un lenguaje sencillo y concreto que favoreció la comprensión de la información, aclarando y repitiendo los conceptos cuando fuera necesario, por tanto se ha visto en estudios anteriores que al repetir varias veces y repasar temas anteriores, se comprende y asimila mejor la información, lo cual fue identificado como positivo por los participantes ${ }^{28}$

Está ampliamente demostrado que las intervenciones en grupos pequeños de entre 8 y 10 personas tienen mayor impacto que la tradicional (individual), la comunicación interactiva permite el intercambio de experiencias y refuerzan el empoderamiento, la participación y satisfacción de las personas ${ }^{29,32-34}$.

Nuestro estudio refuerza lo encontrado por Ramírez, et al.35, quienes demostraron que utilizar la metodología grupal activa participativa permitió a las personas con diabetes y Pre-D cambiar su actitud (de un rol pasivo a uno activo) y ser responsables de su propia enfermedad por medio de los conocimientos adquiridos (saber conocer, saber hacer y saber ser).

Tejada, et al..$^{36}$ encontraron que las personas con diabetes consideran que el apoyo familiar es efectivo cuando los integrantes del núcleo están pendientes de ellos y realizan actividades en conjunto, pues los hace sentir bien y capaces de afrontar su padecimiento con más tranquilidad. La integración de acompañantes (especialmente familiares) en las 
sesiones del PINEC constituyó la principal fuente de apoyo social. La asistencia y el brindar recomendaciones enfocadas al núcleo familiar se puede asociar a motivación, a actitudes más positivas y a un mejor afrontamiento de la enfermedad, lo cual disminuye el estrés generado por la patología, aspectos observados en otras investigaciones $37-40$.

La intervención educativa de PINEC contribuyó a que las personas participantes de este proceso tomaran la responsabilidad de su enfermedad y esto se refleja en el estudio de Roselló-Araya, et al. ${ }^{41}$, en el cual se logró una disminución significativa en la hemoglobina glicosilada pasando de $8.76 \pm 2.14$ a $7.81 \pm 1.78 \%$, en la glicémia en ayunas $(173.3 \pm 76$ a $152.3 \pm 63.47 \mathrm{mg} / \mathrm{dl})$ y en la glicémia posprandial (181.7 \pm 79.9 a $161.7 \pm$ $71.9 \mathrm{mg} / \mathrm{dl})$, en los triglicéridos (247.5 \pm 192.88 a $220.9 \pm 185.1 \mathrm{mg} / \mathrm{dl}$ ) y en la circunferencia abdominal una reducción de 1.1 centímetros.

Respecto con las variables dietéticas, se dio un ajuste en los tiempos de comida, especialmente en las meriendas, mejoró la distribución diaria de los carbohidratos y aumentó el consumo de leguminosas, frutas, vegetales y alimentos fuentes de proteínas. Todo lo anterior evidencia cambios en el control metabólico y este estudio cualitativo refuerza el impacto del PINEC en los objetivos terapéuticos propuestos.

Los resultados del PINEC coinciden con estudios que demuestran los beneficios obtenidos con este tipo de intervenciones educativas, donde se han observado disminuciones significativas en el índice de masa corporal, perímetro de cintura, glicémia en ayunas, glicémia posprandial, colesterol total, presión arterial, triglicéridos y hemoglobina glicosilada, confirmando que estas estrategias son favorables en el control metabólico de la persona con diabetes 33,42-44.

A pesar de que estos estudios mencionados evalúan la efectividad de las intervenciones en pacientes con diabetes, sobre todo su efecto en los objetivos terapéuticos, se carece de estudios donde se explore la intervención educativa como tal. Por lo tanto, la técnica cualitativa de grupos focales utilizada en este estudio permitió conocer el sentir de quienes participaron sobre las sesiones educativas, el material didáctico, contenido temático, metodología grupal y el rol de la persona facilitadora y se demostró que el programa tuvo buena aceptación en la población objetivo.

De acuerdo con los resultados obtenidos y con la experiencia evaluada, se concluye que el PINEC tiene impacto en el control metabólico de la enfermedad y por ser el único programa estructurado de intervención educativa en el país, se tomó como base para la atención y enseñanza de las personas con diabetes y Pre-D en el sistema de salud público de Costa Rica.

\section{AGRADECIMIENTOS}

Al Lic. Miguel Cetina por la recolección de la información y a las personas con diabetes y Pre-D que participaron en los grupos focales.

\section{FUENTES DE FINANCIAMIENTO DE LA INVESTIGACIÓN}

Este trabajo se realizó en el marco del convenio cooperación INCIENSA-CCSS. Fue financiado con fondos públicos del Gobierno de Costa Rica. Las autoras no tienen ningún conflicto de interés.

\section{BIBLIOGRAFÍA}

\footnotetext{
1. Vigilancia de los factores de riesgo cardiovascular [Internet]. San José, Costa Rica: Caja Costarricense de Seguro Social, Editorial Nacional de Salud y Seguridad Social; 2011 [fecha de consulta: octubre de
}

.


2018]. Disponible en: http://www.binasss.sa.cr/informesdegestion/ vigilancia.pdf

2. Vigilancia de los factores de riesgo cardiovascular. Segunda encuesta, 2014[Internet]. San José, Costa Rica: Caja Costarricense de Seguro Social, Editorial Nacional de Salud y Seguridad Social; 2014. Disponible en: http://www.binasss.sa.cr/informesdegestion/encuesta2014.pdf

3. Fernández A, Abdala TA, Alvara EP, Tenorio GL, López E, Cruz S, et al. Estrategias de autocuidado en pacientes con diabetes mellitus tipo 2. Rev Esp Méd Quir. 2012;17(2):94-9.

4. Miyar L, Zanetti ML, Daguano M. El conocimiento del paciente diabético sobre su enfermedad, antes y después de la implementación de un programa de educación en diabetes. Rev Latino-am Enfermagem. 2008;16(2):231-7.

5. García R, Suárez R. La educación a personas con diabetes mellitus en la atención primaria de salud. Rev Cubana Endocrinol. 2007;18(1).

6. Yoldi C. Formación en educación terapéutica en diabetes. ¿Qué tenemos y qué nos falta? Av Diabetol. 2011;27(3):100-5.

7. Diabetes [Internet]. Ginebra: Organización Mundial de la Salud; 2016. Disponible en: https://www.who.int/diabetes/es/

8. Aráuz AG, Guzmán S, Padilla G, Roselló M, Cartín F, Yung G, et al. Evaluación de un programa educativo en diabetes para el nivel primario en salud. Rev Costarric Salud Pública. 2008;17(32):10-6.

9. Kattah W, Coral P, Méndez F. Evaluación del impacto de un programa de tratamiento y educación en la reducción de los niveles de hemoglobina glucosilada en pacientes diabéticos. Act Méd Colomb. 2007; 32(4):206-11.

10. Alarcón NS, Rizo MM, Cortés E, Cadena F. Autocuidado y adherencia terapéutica en diabetes desde una perspectiva del paciente. Waxapa. 2012;2(7):24-32.

11. Mendoza MA, Velasco JF, Nieva RN, Andrade H, Rodríguez CV, Palou $\mathrm{E}$, et al. Impacto de un programa institucional educativo en el control del paciente diabético. Rev Med Inst Mex Seguro Soc. 2013;51(3):254-9.

12. Aráuz AG, Sánchez G, Padilla G, Fernández M, Roselló M, Guzmán S. Intervención educativa comunitaria sobre diabetes en el ámbito de la atención primaria. Rev Panam Salud Pública/Pan Am J Public Health. 2001;9(3):145-53.

13. Funnell MM, Brown TL, Childs BP, Haas LB, Hosey GM, Jensen B, et al. National standards for diabetes self-management education. Diabetes Care. 2011;34(1):S89-96.

14. American Diabetes Association. Standards of Medical Care in Diabetes2014. Diabetes Care [Internet]. 2014;37(1):S14-S80. Disponible en: http:// care.diabetesjournals.org/content/37/Supplement_1/S14.full-text.pdf

15. Figuerola D, Bosch M, Cabasés T, Cabré JJ, Coma C, Flores M, et al. Manual de educación terapéutica en Diabetes. España: Díaz de Santos; 2010.

16. Argudín Y. Educación basada en competencias. Nueva Época. 2001;16:1-29.

17. Cepeda JM. Metodología de la enseñanza basada en competencias. Rev Iberoam de Educ. 2004;35(1):1-10.

18. Aráuz AG, Guzmán S, Roselló M, Brenes M, Solano I, et al. Informe técnico: Programa de intervención nutricional en enfermedades crónicas, modelo de atención en diabetes mellitus. Tres Ríos, Costa Rica: INCIENSA, 2013.

19. Castillo A, Ferrer L, Masalán P. Capacitación del personal de salud, evidencia para lograr el ideal. Horiz Enferm. 2015;26(1):29-37.

20. Martínez C. El muestreo en investigación cualitativa. Principios básicos y algunas controversias. Ciência \& Saúde Coletiva. 2012;17(3)613-9.

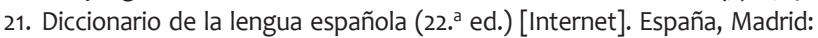
Real Academia Española; 2001. Disponible en http://www.rae.es/rae.html

22. Hevia P. Educación en diabetes. Rev Med Clin Condes. 2016;27(2):271-6.

23. Pereira L, Antunes E. "El grupo facilita todo": significados atribuidos por pacientes con diabetes mellitus tipo 2 a grupos de educación para la salud. Rev Latino-Am Enfermagem. 2014;22(6):980-7.

24. Trento M, Passera P, Borgo E, Tomalino M, Bajardi M, Cavallo F, et al. A 5-year randomized controlled study of learning, problem solving ability, and quality of life modifications in people with type 2 diabetes managed by group care. Diabetes Care. 2004;27:670-75.

25. Pimentel JA, Sanhueza O, Gutiérrez JM, Gallegos EC. Evaluación del efecto a largo plazo de intervenciones educativas para el autocuidado de la diabetes. Cienc Enferm. 2014;20(3):59-68.

26. García R, Suárez R. Resultados de la estrategia cubana de educación en diabetes tras 25 años de experiencia. Rev Cubana Salud Pública. 2007;33(2).

27. García R, Suárez R, Mateo-de-Acosta O. Comunicación y educación interactiva en salud y su aplicación al control del paciente diabético. Rev Panam Salud Pública. 1997;2(1):32-6.

28. De Dios JA, Jiménez ME. La comunicación en salud desde las perspectivas ética, asistencial, docente y gerencial. MEDISAN. 2009;13(1).

29. Alarcón NS, Cadena F, Reyes A, Guajardo V. Intervención educativa de salud en pacientes con diabetes mellitus para fortalecer el apego al tratamiento. Waxapa. 2012;1(6):31-7.

30. Bolaños E, Sarría A. Perspectiva de los pacientes sobre la diabetes tipo 2 y relación con los profesionales sanitarios de atención primaria: un estudio cualitativo. Aten Primaria. 2003;32(4):195-202.

31. Rodrigues FFL, Zanetti ML, Santos MA dos, Martins TA, Sousa VD, Teixeira CR de S. Knowledge and attitude: important components in diabetes education. Rev Latino-Am Enfermagem. 2009;17(4):468-73.

32. Arcega A, Celada NA. Control de pacientes con diabetes: Impacto de la educación participativa versus educación tradicional. Rev Med Inst Mex Seguro Soc. 2008;46(6):685-90.

33. Cabrera CE, González G, Vega MG, Arias ED. Impacto de la educación participativa en el índice de masa corporal y glucemia en individuos obesos con diabetes tipo 2. Cad Saúde Pública. 2004;20(1):275-81.

34. Steinsbekk A, Rygg L, Lisulo M, Rise MB, Fretheim A. Group based diabetes self-management education compared to routine treatment for people with type 2 diabetes mellitus. A systematic review with meta-analysis. BMC Health Serv Res. 2012;12(213):1-19.

35. Ramírez A, Aráuz AG, Guzmán S, Roselló M. Conocimientos, prácticas y percepciones sobre la enfermedad de personas con diabetes $y$ prediabetes después de participar en un programa educativo grupal. Rev ALAD. 2016;6(1):7-17.

36. Tejada LM, Pastor MP, Gutiérrez SO. Efectividad de un programa educativo en el control del enfermo con diabetes. Invest Educ Enferm. 2006; 24(2):48-53.

37. Azzollini S, Bail V, Vidal V. El apoyo social y el autocuidado en diabetes tipo 2. Anuario de Investigaciones. 2012;19:109-13.

38. Dos Santos MA, Alves RCP, de Oliveira VA, Ribas CRP, Texeira CRS, Zanetti ML. Representações sociais de pessoas com diabetes acerca do apoio familiar percebido em relação ao tratamento. Rev Esc Enferm. USP. 2011; 45(3):651-8.

39. Martínez B, Torres LE. Importancia de la familia en el paciente con diabetes mellitus insulinodependiente. Psicología y Salud. 2007;17(2): 229-40.

40. Gonzáles P. El apoyo familiar en la adherencia al tratamiento nutricional del paciente con diabetes mellitus tipo 2. Waxapa. 2011;2(5):102-7.

41. Roselló-Araya M, Guzmán S, Aráuz AG, Arias D, Schmidt M, Vargas M. Efecto de la metodología de educación terapéutica, mediante la evaluación de los cambios bioquímicos, antropométricos, dietéticos en las personas con diabetes mellitus tipo 2. ALAD. 2018; 8:99-109.

42. León-Mazón M, Araujo-Mendoza G, Linos-Vázquez Z. Eficacia del programa de educación en diabetes en los parámetros clínicos y bioquímicos. Rev Med Inst Mex Seguro Soc. 2013;51(1):74-9.

43. López-Portillo A, Bautista-Vidal RC, Rosales-Velásquez OF, Galicia-Herrera L, Rivera-y Escamilla JS. Control clínico posterior a sesiones grupales en pacientes con diabetes e hipertensión. Rev Med Inst Mex Seguro Soc. 2007;45(1):29-36.

44. Vázquez M, Villegas H, Ramírez F, Bolaños E, Granados E, Silva L, et al. Intervención integral en pacientes diabéticos tipo 2. Revista médica de Costa Rica y Centroamérica. 2014;71(612):863-6. 